EPJ Web of Conferences 41, 05033 (2013)

DOI: $10.1051 /$ epjconf/20134105033

(C) Owned by the authors, published by EDP Sciences, 2013

\title{
Femtosecond Electron Diffraction Study of the Cyclization Reaction in Crystalline Diarylethene
}

H. Jean-Ruel ${ }^{1}$, M. Gao ${ }^{1}$, C. Lu ${ }^{1}$, L. Liu ${ }^{1}$, G. Moriena ${ }^{1}$, R. R. Cooney ${ }^{1}$, M. A. Kochman ${ }^{2}$, C. A. Morrison $^{2}$, G. Sciaini ${ }^{3}$, and R. J. D. Miller ${ }^{1,3, a}$

1 Departments of Chemistry and Physics, 80 St. George Street, University of Toronto, Toronto, Ontario, M5S 3H6, Canada

2 School of Chemistry and EaStCHEM Research School, University of Edinburgh, West Mains Road, Edinburgh, UK, EH9 3JJ

3 Max Planck Department for Structural Dynamics, Department of Physics, University of Hamburg, Centre for Free Electron Laser Science, DESY, Notkestrasse 85, Hamburg 22607

\begin{abstract}
Femtosecond electron diffraction is used to directly resolve the atomic motions involved in the ring closing reaction induced in a photochromic single crystal. The measurements were performed using our newly developed setup capable of delivering sub-500 fs electron bunches containing $10^{6}$ electrons per pulse. Theoretical calculations were also performed.
\end{abstract}

\section{Introduction}

Diarylethene derivatives are a family of photochromic molecules which undergo photoinduced reversible isomerization in both the solution and crystal phase in which there is conserved stereochemistry [1]. Here we present the study of the ring closing reaction on one such compound, 1,2-bis(2,4dimethyl-5-phenyl-3-thienyl) perfluorocyclopentene that provides a model system for understanding cyclization reactions mediated through conical intersections.

In order to characterize the timescales involved and to optimize the excitation conditions for the electron diffraction experiment, we have recently performed femtosecond transient absorption studies of the cyclization reaction in this diarylethene derivative in its crystalline phase [2]. After demonstrating the possibility to achieve $3 \times 10^{4}$ repeatable ring closing and opening cycles, the dynamics of interest were fully resolved using a robust acquisition scheme which directly exploits this photoreversibility. It was found that immediately following the excitation, relaxation of the molecule on the open-ring excited state potential energy surface, along the reaction coordinates, occurs on a time scale of approximately $200 \mathrm{fs}$. The ring closing reaction then proceeds from this intermediate with a time constant of 5.3 ps. Those results set the requirements for the time resolution needed for the FED experiment. Here we report our latest results associated to the FED study of this classical electrocyclic reaction. In situ electron diffraction cycling measurements of the diarylethene samples are shown and the capabilities of our FED setup - sub-ps time resolution with electron bunches capable of near single-shot diffraction pattern acquisition - are demonstrated in the context of this molecular system. Theoretical calculations are also presented.

\footnotetext{
a e-mail: dwayne.miller@mpsd.cfel.de
} 


\section{Experimental Methods}

The electron diffraction measurements presented below are conducted with a $100 \mathrm{kV}$ electron gun combined to a RF compression system capable of delievering sub-ps electron bunches containing $1 \times 10^{6}$ electrons per pulse. These pulses have been fully characterized using a cross correlation pondermotive scattering with the laser excitation. For diarylethene, the pump pulses used to induce the ring closing reaction are centered at $270 \mathrm{~nm}, 70 \mathrm{fs}$. The specificity of diarylethene further requires that

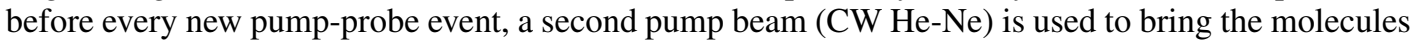
back to their initial state (ring-open), since the closed-ring form is also thermally stable. The photochromicity of diarylethene presents in fact a great advantage for time-resolved study compared to samples that recover thermally, since the $\tau_{\infty}$ points for each pump-probe event can be recorded in order to systematically monitor the reversibility conditions of the structural changes. The crystals were microtomed to $100 \mathrm{~nm}$ thick and mounted on TEM copper grids held at room temperature.

\section{Results and Discussion}

The reversibility of the structural changes associated with subsequent ring closing and opening in crystalline diarylethene induced with such excitation conditions was directly investigated with electron diffraction. Figure 1(a) shows a schematic of the isomerization reaction. Figure 1(b) shows the differential diffraction pattern between the closed- and open-ring states (using 5\% excitation), corresponding respectively to the $\tau_{-\infty}$ and $\tau_{\infty}$ points of a time-resolved experiment. It can be observed that a large number of Bragg peaks change intensity, unambiguously demonstrating our capability to observe the structural changes involved in the photoinduced ring-closing reaction. Figure 1(c) shows the relative change in intensity of the diffraction peaks circled in Figure 1(b) as the sample is cycled between the closed- and open-ring states. Although more than $3 \times 10^{4}$ repeatable cycles were achieved with the excitation conditions of the transient absorption measurements, as mentioned above, it can be observed that only approximately $3 \times 10^{2}$ repeatable cycles - considering a $80 \%$ criteria - are achieved with the pump conditions required for the FED experiment. The reason is that for the transient absorption measurements, the unexcited molecules are effectively transparent to the probe, such that thick crystals and very low fractional excitation can be used. In FED, on the other hand, the mean free path of the electrons into solid state samples is on the order of $100 \mathrm{~nm}$, such that ultrathin crystals and very high fractional of excitation are needed. This limited number of reversible cycles imposes strong restrictions on the acquisition conditions required for a time-resolved experiment. In order to achieve the SNR needed to accurately follow the structural changes associated to the ring closing reaction, the system should acquire near single-shot diffraction patterns. The inset of Figure 1(c) shows a typical grating enhanced ponderomotive scattering trace for electron bunches containing $1 \times 10^{6}$ electrons [3]. The present time resolution results predominantly from a combination of the actual individual electron bunches time duration and the shot-to-shot jitter. This trace thus represents the instrument response function of the FED setup, demonstrating sub-400 fs temporal resolution. Such resolution is capable of resolving sub-ps and ps atomic motions involved in the ring closing reaction of crystalline diarylethene. Furthermore, considering that the acquisition of a diffraction pattern for such complex organic molecules containing no heavy atoms requires approximately $10^{7}$ electrons, the system is near the single-shot diffraction pattern acquisition limit.

Theoretical calculations have also been performed to assist the assignment of the time-resolved diffraction data. A model for the cyclization reaction intermediate was constructed using ab initio calculations which include a novel hybrid method in which CASSCF and DFT levels of theory are combined to generate a potential energy surface for an excited diarylethene molecule embedded in a crystal lattice. The optimized structure is shown in Figure 1(d). These theoretical calculations will help identify the key motions involved in the reaction pathway.

The FED data collection is currently in progress. A large number of samples with different orientations have already been measured, several of which exhibit ultrafast changes in diffraction intensity 
a)
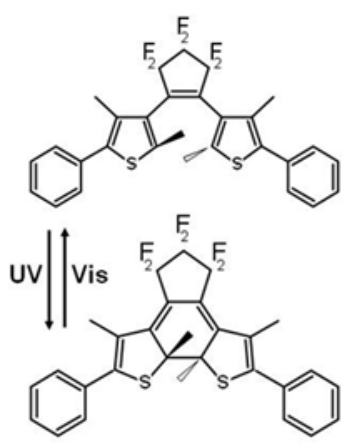

c)

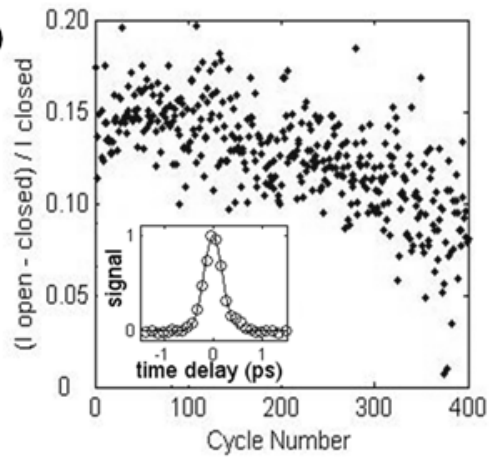

b)

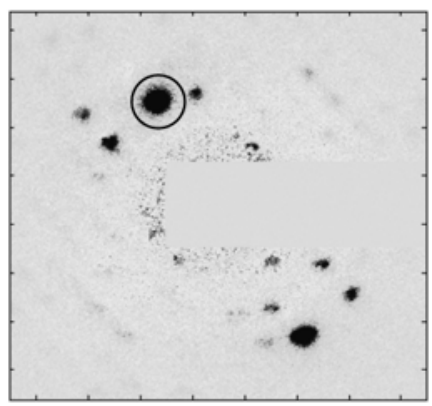

d)

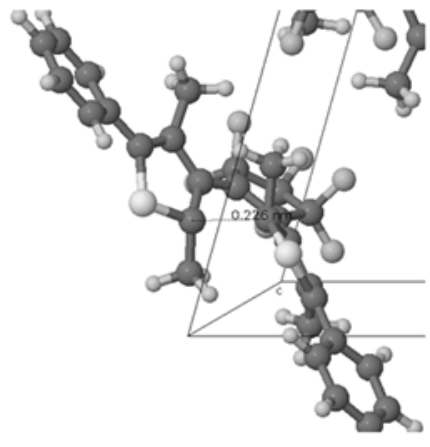

Fig. 1. (a) Schematic of the ring closing reaction. (b) Differential electron diffraction pattern between the closedand open-ring states (5\% excitation). (c) Relative change in integrated intensity of a selected diffraction peak between the open-ring and closed-ring state for the first 400 cycles. It is observed that following the cyclization reaction the diffraction intensity initially changes by $15 \%$. However, after 400 such ring closing and opening cycles, the relative change is down to only $10 \%$ due to optical fatigue. The inset shows a grating enhanced ponderomovive scattering trace of the electron bunches. (d) Calculated optimized geometry for the intermediate state of the cyclization reaction of diarylethene embedded in the crystal lattice.

with dynamics in agreement with the findings of the transient absorption study. Slower dynamics (100 ps time scale) associated to strain effects and acoustic phonons are also observed. When the data collection will be completed, the direct reconstruction of the atomic motions associated to the ring-closing reaction in crystalline diarylethene should be possible.

\section{References}

1. M. Irie, Chem. Rev. 100 1685, (2000)

2. H. Jean-Ruel et al., J. Phys. Chem. A. 115(45) 13158, (2011)

3. C. T. Hebeisen et al., Optics Express 16 333, (2008) 